\title{
Responding to the mental health consequences of the 2015-2016 terrorist attacks in Tunisia, Paris and Brussels: implementation and treatment experiences in the United Kingdom
}

\author{
ABSTRACT \\ Objectives: To explore whether the Screen and Treat Programme to support United Kingdom \\ citizens potentially affected by terrorist attacks in Tunisia (2015), Paris (2015) and Brussels (2016) \\ was effective in identifying and referring people to mental health services, to examine the \\ programme's acceptability to users and to understand how agencies involved worked together. \\ Methods: Individuals offered screening by the programme $(n=529)$ were invited to participate in \\ the study and were sent a questionnaire. Follow-up interviews were conducted with questionnaire \\ respondents who consented and with employees of agencies involved in the programme's \\ planning and delivery. Seventy-seven people affected by the attacks completed questionnaires, 35 \\ of those were also interviewed, and one further person only participated in an interview. Eleven \\ people from agencies organizing and delivering the programme and five clinician-managers were \\ also interviewed. \\ Results: Most service users said the attacks had a major impact on their lives. Many reported \\ anxiety, depression, difficulty going out or travelling, sleep problems, panic attacks, flashbacks and \\ hyper-vigilance. A third had reduced their working hours and a similar proportion had taken sick \\ leave. Two-thirds sought help from their GP before being contacted by the programme, but almost \\ all thought their GP had not been helpful in dealing with post-traumatic stress disorder (PTSD) or \\ referring to appropriate care. Several people were prescribed psychotropic medication; only a few \\ were referred to mental health professionals. Many participants used help offered by \\ organisations external to National Health Service (NHS), with mixed experiences.
}


Waiting times for treatment varied from no delay to a few months. Most interviewees thought the Programme should have started sooner and provided more information about sources of support. Most users found treatment received via the programme helpful.

Professionals involved in organizing and delivering the programme thought that bureaucratic delays in setting it up were key limitations on effectiveness. Clinician interviewees thought an outreach approach was needed to identify at-risk individuals.

Conclusions: Users who took part in the programme were satisfied with their treatment, although many thought it should have been offered sooner. Funding and data sharing between agencies were the main barriers to timely contact with affected individuals. Self-referral, GP identification of PTSD and GP referral to appropriate care were regarded as ineffective, suggesting that people affected by similar future incidents should be supported better and assisted more promptly to access treatment.

\section{Background}

Terrorist attacks are frequent global phenomena with wide-reaching health and other consequences. Post-traumatic stress disorder (PTSD) and other mental health problems are common concerns for those affected, $[1,2]$ but often missed by healthcare professionals[3]. Selfreferral and use of routine medical care appear to be low[4], leading to unmet mental health needs.

There is limited experience in establishing effective mental health service responses for individuals affected by terrorist attacks. Although some people recover on their own, many need support to access timely, effective treatment.[5] Several guidelines describe evidence-based approaches to providing mental health support and treatment for people affected by terrorist attacks, [6-8] but many have not been implemented in practice. $[9,10]$ 
The Screen and Treat Programme was established by the then Department of Health (DH) in England in March 2016 to support United Kingdom (UK) citizens affected by terrorist attacks in Tunisia (March and June 2015), Paris (November 2015) and Brussels (March 2016). Questionnaires screening for PTSD (Trauma Screening Questionnaire; TSQ)[11], anxiety and depression (Patient Health Questionnaire-4; PHQ-4)[12], and alcohol use (Alcohol Use Disorders Identification Test (AUDIT-C)[13] were sent to approximately 500 people (injured, bereaved or witnesses) by the Metropolitan Police and returned to Public Health England (PHE). People who screened positive were assessed by the Psychological Response to Traumatic Events (PRTE) team at South London and Maudsley NHS Foundation Trust. People in need of treatment were referred to local mental health services, such as Improving Access to Psychological Therapies (IAPT), and followed up by PRTE until end of their care.

Outcomes of the screening part of the programme suggest that it was generally successful in facilitating access to services.[14] Of the 195 people who completed screening, 179 (91.8\%) screened positive on one or more indicators measuring PTSD, anxiety, depression, increased smoking, or problematic alcohol consumption. After clinical assessment, 78 adults were referred for treatment.[14]

\section{Aims of the evaluation}

The aims of this evaluation were to explore whether the Screen and Treat Programme was effective in identifying and referring people to appropriate services, to test the programme's acceptability to users, and to understand how agencies involved worked together. 


\section{Methods}

\section{Procedure}

Between September and November 2016, 529 adults affected by the incidents were invited to participate in the evaluation. Most (476) had been in Tunisia, 41 in Paris and 12 in Brussels.

Potential user participants were sent a letter, via the $\mathrm{DH}$, with information sheet, consent form, questionnaire and stamped pre-addressed envelope. Responders who consented to being interviewed were contacted by a researcher. Interviews were conducted by telephone $(n=33)$ or face-to-face $(n=3)$.

A total of 78 responded -42 completed the questionnaire, 35 completed the questionnaire and interview and one completed the interview only. The response rate was 15 per cent. Ninety-one per cent of respondents had been in Tunisia, four per cent in Paris and three per cent in Brussels (the remainder did not indicate where they had been). Sixty-three per cent of user respondents had taken part in the programme, 27 per cent did not. The remainder did not indicate whether they had taken part.

To protect user participant anonymity, the results below do not report respondents' incident location, as very few UK citizens were affected by the Paris and Brussels incidents, and even fewer took part in the evaluation.

Representatives of agencies involved in setting up and delivering the programme were contacted by email and invited to participate in telephone interviews conducted between October 2016 and March 2017 by a researcher. Eleven employees of agencies involved in planning and delivering the programme (DH, PHE, PRTE, NHS England, Metropolitan Police) were interviewed. Twelve clinicians from service providers who had received multiple referrals via the programme were invited to participate; of those, five clinician-managers were interviewed. 
All participants provided written consent. Detailed electronic notes were taken during interviews.

\section{Questionnaire}

The self-completion questionnaire was based in part on a modified Client Service Receipt Inventory (CSRI)[15] with additional questions about impact of the event on work, studies, family responsibilities and other activities.

\section{User and professional interviews}

Interview topic guides were informed by National Institute for Health and Care Excellence (NICE) Guidance on treatment of PTSD[16]; previous studies[4, 17-19]; and consultation with PTSD experts and an expert-by-experience.

Service users were asked about their experiences of the programme, including screening, assessment and referral; information provided to them; symptoms; health status; time delays and their decision (or not) to engage with the programme. They were also asked the timing of the programme and any delays they experienced; about the treatment options they were offered and if they were satisfied with them; and about their overall satisfaction with the programme, what worked well for them and what could be improved.

Interviews with professionals organizing and delivering the programme asked about the process of providing responses, including information sharing between agencies, how users were moved through the programme and impact of the programme on local services due to the additional individuals affected by terrorism.

\section{Analysis}

Based on issues identified in the literature[20], an initial analysis framework was created, tested on six interviews and revised. Data were analysed using the revised framework. Any new topics were noted during revision and subsequent analysis phases. 


\section{Ethical review}

The Health Research Authority classified this project as a service evaluation not requiring NHS

Research Ethics Committee review or NHS R\&D approvals. The study underwent ethics review in keeping with the London School of Economic and Political Science's Research Ethics Policy and Procedures.

\section{Results}

\section{Characteristics of users}

Table 1 gives numbers of users who completed evaluation questionnaires and interviews and their engagement with the programme. Table 2 shows users affected by each incident.

Table 1. Number of participants in each evaluation element (as of March 2017)

\begin{tabular}{|l|r|r|r|r|}
\hline & \multicolumn{3}{|c|}{ Participation in Screen and Treat Programme } & Total \\
& \multicolumn{2}{|c|}{$(\mathrm{N})$} & \\
\hline Evaluation element & Took part & $\begin{array}{l}\text { Did not take } \\
\text { part }\end{array}$ & $\begin{array}{l}\text { Did not } \\
\text { indicate }\end{array}$ & \\
\hline Interview and questionnaire & 28 & 7 & - & 35 \\
\hline Questionnaire only & 21 & 13 & 8 & 42 \\
\hline Interview only & - & 1 & - & 1 \\
\hline Total & 49 & 21 & 8 & 78 \\
\hline
\end{tabular}

Table 2. Numbers of participants affected by each incident

\begin{tabular}{|l|c|c|c|c|}
\hline & \multicolumn{3}{|c|}{ Incidents } & \multirow{2}{*}{$\begin{array}{c}\text { Did not } \\
\text { indicate }\end{array}$} \\
\hline Questionnaire responders & Tunisia & Paris & Brussels & 2 \\
\hline Interview responders & 70 & 3 & 2 & 2 \\
\hline
\end{tabular}

\section{Impact of the attacks}

The following reports users' responses to questionnaire and interviews. The number of respondents varies as most participants only responded to some questions. 


\section{Onset of mental health problems}

Two-thirds of questionnaire respondents ( 46 of 67 ) reported that daily activities, such as housework and family responsibilities, had been adversely affected. Most interviewees (31 of 36) reported that, since the incident, they had been unable to get on with their lives as before. However, five people said that the event had relatively limited impact:

I learned to live with it, tried to get on with my life as soon as possible, shut the door on it. It will never go away. The news brings it back. We lost two friends, but we still got to live, you can't let things like this dictate you.

Of the 12 interviewees who answered a question about timing of their symptoms, seven realized immediately after the incident that they were experiencing mental-health problems, while four reported a delay in symptom onset:

[Three months later] in a restaurant I had an extreme startle response (to party poppers). I was hiding under the table. It took me days to calm down. Then the episodes increased.

Anxiety, panic attacks and PTSD symptoms (re-experiencing the incident, avoidance, hypervigilance) were the most frequently-reported effects. Several questionnaire respondents were afraid to go out or were constantly on high alert, and six reported difficulty travelling or going on holiday. A third of the interviewees experienced acute anxiety in public places, and so avoided going out. Several found it difficult to cope with loud noises, especially fireworks.

Nine respondents reported sleep problems, nightmares and flashbacks. Many reported low mood, depression, lack of motivation, difficulty concentrating and social isolation:

I can't be bothered, have no inclination to do anything or go anywhere.

A few people developed preoccupations and spent a lot of time thinking about how close they had come to being killed; some had friends or relatives who had been killed in the attack: 
I lost three family members in the Tunisia beach attack. I find it very difficult to accept the way they died. I live day to day and I find life very angry, I don't bother with people, only family. I find it hard to accept our loss.

Several respondents had experienced relationship difficulties since the attack:

I argue with [my] husband and distance myself from family members for fear of [them] dying.

\section{Physical health}

Physical injuries sustained in the attacks had left some disabled:

Initially, I was discharged from hospital, unable to do housework. I can't leave the house unless in a wheelchair.

Four interviewees (none of whom were injured) reported significant deterioration in their physical health as a result of the attack (e.g. multiple sclerosis deteriorating) and one was disabled as a result of the attack.

\section{Impact on work, studies and finance}

Over a third of questionnaire respondents (22 of 58) had reduced their working or studying hours, 24 of 66 had taken sick leave and 7 of 61 had become unemployed or had ended/interrupted their studies. Time taken off work varied from a few days to several months.

Before the incidents, 28 of 36 interviewed had been in regular work, six retired and two not working. Following the attacks, 13 people took time off work, ranging between two weeks and 10 months. A few did not take time off work, finding it harder being at home:

In the end I had four months off work. I was not able to function, then I had a phased return to work. It took all year.

Four people had financial concerns, in addition to the trauma they experienced: 
Loss of income, lost financial security and [loss of a] loving husband. I was unable to return to my role at work. I'm just returning on a long-term phased plan with different responsibilities.

\section{Use of support services}

By the time of receiving the screening questionnaire, 46 of 74 users had already seen an NHS psychologist or counsellor, and half (32 of 62 ) had seen a private therapist. Fewer (12 of 66) had seen a nurse, been to an A\&E department (8 of 69) or seen an NHS psychiatrist (6 of 61).

Interviewees varied considerably in their desire for help and support:

It wasn't until I got the referral from government offering help...Some people don't always ask for help...it would be good to have some information available. I was worried that I would be sectioned, how severe it was...People don't realize that it is a normal reaction, you don't need a straitjacket.

\section{Experiences with GPs}

Nearly two-thirds of questionnaire respondents (47 of 74) had seen their GP as a result of the attack. Of the 36 users interviewed, 25 sought help from their GP before the programme's launch, but only nine thought their GP had been helpful or sympathetic. GPs appeared more willing to deal with physical symptoms (e.g. minor injury, heart problems, asthma) than mental-health problems:

[My GP] was a bit shocked, she didn't say a lot and offered tablets...She didn't know what to tell me.

Thirteen people were prescribed sleep and/or antidepressant medication. Only two people said the medication helped; most did not take it.

\section{GP referrals to mental health support}

Of the 25 interviewees who sought help from their GP, eight people were referred to a mentalhealth professional (e.g. mental health nurse, counselling or generic cognitive behavioural therapy), but seven people found the treatment did not help: 
I went to the GP, got sleeping pills and was sent to a nurse. She was not able to deal with it.

Interviewees from agencies organizing the programme were surprised that GPs did not recognize PTSD:

My idea of a GP is naïve. I would expect referral [to mental health services] automatically when they hear the word 'terrorist'.

\section{Hospital admissions and outpatient attendances}

Six of the 77 questionnaire respondents were admitted to hospital with physical injuries or medical problems as a result of the incident. Reasons for admission included gunshot wounds and angina. Total time spent as a hospital inpatient ranged between three and 108 days.

Fifteen questionnaire respondents said they had been referred to a hospital as an outpatient. Number of outpatient appointments ranged between 1 and 50.

\section{Non-NHS support}

About a third of questionnaire respondents received support from non-NHS organisations, with mixed experiences, including counselling offered by the travel agent; support or therapies offered by charities such as Victim Support, Assist Trauma Care and Samaritans; unregistered therapists and professional help arranged by employers. Most of the 14 user interviewees who used counselling provided by the travel agent did not find it helpful. Six interviewees praised the Foundation for Peace/Survivors' Assistance Network for supporting them to get appropriate help:

It was well over a year before I got some help. The most helpful were the Foundation for Peace who wrote a letter to the GP and got some further help.

Several people said the Facebook group set up by one survivor was very helpful. Many participants said that lack of mental health support immediately after the incident meant they looked for any help available. 


\section{Experiences of Screen and Treat Programme}

\section{Timing}

Of the 36 user interviewees, 28 accessed the programme, and 26 of those expressed views about its timing. Twenty-one thought the programme started too late and that their health deteriorated before they received treatment. Many felt that access to support earlier would have allowed them to improve earlier:

[By the time the programme was available] it was too late, I had already decided I couldn't cope with that job. I couldn't go back to work at the end of October. This [the programme] started in April. Shame it was too late.

\section{Screening and assessment}

Users consistently praised the PRTE team for regular contact, care coordination with local services and telephone support while awaiting treatment:

[The psychologist] was very supportive. [She] rang back on a monthly basis, to check how things are progressing. She also spoke to the lady who is doing EMDR [eye movement desensitization and reprocessing].

All professional interviewees thought the outreach worked well and that, without it, people would have missed the opportunity to receive appropriate care:

[People affected by these traumatic experiences] do not think of themselves as having mental health problems and that makes them reluctant to come forward.

Some professionals questioned the value of the screening process, as almost all screening results were positive.[9] However, they thought it would have been difficult to coordinate the programme without the central PRTE team. Clinicians described the PRTE's clinical assessments as very useful. 


\section{Waiting times}

Most user questionnaire respondents (61 of 77) had been seen within 18 weeks ${ }^{1}$ of referral, while 16 waited longer than 18 weeks for their first appointment. Three interviewees said that PRTE had needed to put pressure on local services as they were not getting treatment even after several months' wait. During that time, one user had felt "isolated and not cared about". Another described difficulties caused by waiting for treatment:

I spent four months waiting for an appointment. That was the hardest, it did a lot of mental harm.

Service-provider interviewees reported average waiting times ranging between two and 25 weeks. Some services were over-subscribed, resulting in long waiting lists. Although the instruction from NHS England was not to prioritize people affected by the attacks ahead of other patients, some IAPT services gave them higher priority. Conversely, other services did not start treatment within the 18-week waiting target.

\section{Treatment}

Service provider interviewees said that PRTE was crucial for the effective running of the programme's treatment stage. The PRTE team liaised with each local therapist, providing a handover and assessment compatible with IAPT requirements. The team offered support, training and supervision to therapists, which was well received. Therapists thought that PRTE made sure that users stayed engaged with services and provided them with useful information and coping strategies:

The ongoing telephone support from the [PRTE] psychologist was incredibly helpful and it gave [the user] a good understanding of coping strategies, which helped our work.

\footnotetext{
${ }^{1}$ The stated target is that $75 \%$ of new referrals to the IAPT programme should begin treatment within six weeks of referral, and 95\% within 18 weeks (The Mandate: A mandate from the Government to NHS England: April 2015 to March 2016, p 16-17, available at: https://www.gov.uk/government/uploads/system/uploads/attachment_data/file/38622 1/NHS_England_Mandate.pdf)
} 


\section{Self-reported treatment outcomes}

Just under a quarter of users who completed the questionnaire thought the treatment they received had helped them stay in work/study or return to work/study earlier than would otherwise have been the case. This was slightly higher for those who had taken part in the programme; nearly a third reported a positive impact of treatment on their work/study.

Overall, the 28 interviewees who took part in the programme were satisfied with the support received. Eighteen users were referred for treatment but ten did not progress beyond screening. Of those referred, 13 were referred for trauma-focussed cognitive behavioural therapy (CBT) and two for eye movement desensitization and reprocessing (EMDR); three were waiting for treatment to start when interviewed. By the time of interview, four people out of 18 had completed treatment, ten were still in treatment and one had withdrawn. Of those ten who did not progress beyond screening, five were already in treatment elsewhere, three did not need referral, and two declined referral.

At the time of interview, four people had completed treatment and were managing well. Each of the 18 interviewees who took up treatment via the programme reported getting worse before treatment started. Subsequently, as a result of the programme, 11 felt they were getting better, one felt the same as before, two were not sure if it was helping, one did not find the treatment helpful and withdrew after four sessions; three were still waiting for treatment to start. Eight of the 10 interviewees still in treatment when interviewed were finding it helpful:

I am almost recovered, almost as normal as before. That would have not happened without Screen and Treat. 


\section{Funding}

The professional interviewees said it was difficult to plan and set up the programme, mainly due to a Governmental commitment to deliver the programme before funding had been identified. Another challenge was estimating the numbers of people who might be eligible and would choose to access the programme, making it difficult to contract a service:

It was difficult to know who to include as other attacks happened during the planning stage [Paris]".

\section{Set-up phase and timing}

Professionals planning and setting up the programme were not surprised by the cumbersome setup, which led to delays:

We were slow off the mark in terms of contacting people...People said they could have done with that six months earlier...It was a year after Tunisia.

Several said that people would have received a better service if it had been embedded in the NHS rather than managed from central government. All professional interviewees thought the delay in setting up the programme had considerably limited its effectiveness, and clinicians were concerned about the consequences of lack of timely and appropriate treatment. One professional said "they had a year to get worse and develop bad ways of coping".

\section{Data sharing}

Respondents said a lack of data-sharing agreements between agencies contributed to delays in contacting affected individuals. Only those who had voluntarily identified themselves to the police upon returning to the UK could be contacted, and only via the police, who carried out all communications on behalf of other agencies, including the DH or PHE. It was impossible for other agencies to contact people directly: 
There was the unresolved issue of how we get the initial list of people, how we identify those at risk. Having a list of everyone exposed would have really helped and follow-up could have been managed better.

Some people who might have needed support would have been missed. Two people affected by the Tunisia event confirmed that some of their fellow passengers bypassed the police at airports because they "just wanted to get home".

\section{Suggestions for improving future responses}

Several professionals felt the service should be built into the NHS and that local services should be used (including GPs) to screen people for PTSD. Another suggestion was for quality-assured nonNHS organisations to be used.

Professionals recommended reaching out to people through several channels, including online. They also suggested that group interventions (e.g. if large numbers of people were affected in one locality) could be offered alongside more intensive one-to-one interventions.

One clinician-manager suggested developing a network of trauma centres around the UK in preparation for coordinating responses ahead of similar future incidents. Another clinicianmanager suggested that it would have been useful to be able to refer 'difficult cases' to a national specialist centre, such as PRTE.

\section{Timing}

User interviewees thought that timely access to specialist therapists should be part of future responses:

There was a delay in getting to the right person. I could have been somewhere else by now.

Several users indicated that earlier information about available support would have encouraged them to pursue it and possibly improve their health earlier: 
Had I received the info earlier, it would have prompted me to do something earlier...I didn't realize how affected I was until other things started to pile up.

Professional interviewees' recommendations included 1-3 months of 'watchful waiting' and checking for symptoms for those identified as at risk. Several professionals felt that a future programme should contact potentially affected people much earlier, give them a point of contact, help them normalize the experience, and provide information about "how to look after themselves".

\section{Information and communication}

Users frequently suggested improving information about help and support, and communication with potentially affected individuals. Users did not mind being contacted, but of the 28 people who engaged with the programme, 10 reported lack of information about available help:

The whole process you felt like you have been in the dark, that you are not cared about, that you are not important.

Six user interviewees said that the Facebook group set up by one survivor was their only information source, and some found out about the programme by accident. Some users were aware of data protection issues preventing agencies from contacting people directly:

When I asked why there wasn't a list from the tour operator, [the MP] said "we cannot do that because of data protection". Surely there must be a way to override this, it was a lame excuse.

\section{Discussion}

The terrorist attacks in Tunisia, Paris and Brussels had profound impacts on the lives of people present or affected indirectly. Most user participants reported how daily activities, working lives and general functioning were affected, often considerably. 
Some people did not seek mental health support, but many who consulted their GP found that they were not offered effective treatment. Medication was the first line of treatment offered by many GPs, despite limited evidence of its effectiveness in treatment of PTSD.[16] Many participants sought help from non-NHS sources, with mixed results.

Given lack of appropriate support, users regarded the Screen and Treat Programme as very valuable. They found the central screening and assessment team very helpful, especially in providing support while waiting for local treatment. Treatment options offered to adults who took part in the programme were trauma-focused CBT and EMDR, recommended by NICE.[16] Users were satisfied with treatment, although most thought it should have been available sooner. A study of the response to the 2015 Paris attacks supports the argument that people affected by terrorist attacks should be able to access appropriate healthcare soon after an incident: those involved in the Paris attacks who received specialist trauma support in the first 48 hours reported depression and anxiety less frequently than those with no support.[5]

Funding and data sharing between agencies were seen as the main barriers to starting the programme promptly and to timely contact with affected populations. Data sharing appears to be a recurrent issue in major incident responses, as found in responses to the Indian Ocean tsunami and the 2005 London bombings. $[21,17]$ Our findings confirm previous evidence that people do not mind being contacted about potential help, indicating that official systems over-estimate the public's desire for confidentiality. Self-referral and GP identification of PTSD and referral to appropriate care were largely opportunistic, suggesting that outreach programmes are needed to support people affected by terrorist incidents to access effective, timely treatment, even with the constraints placed on data sharing between agencies. 
The main limitation of this study is absence of information about the total population potentially affected by the attacks. Individuals who participated in the programme (or evaluation) may not be representative of the population affected.

\section{Conclusion}

This evaluation presents an opportunity for relevant agencies to learn from the programme. Its findings are consistent with conclusions from evaluation of the 2005 London Bombings programme,[17] suggesting that future response-planning would benefit from consideration of recurring issues, such as data-sharing constraints, failure of standard referral pathways and reluctance to self-refer.

In future, evaluations should be embedded in the response to major incidents, with the aim of reaching the entire population of potentially affected individuals. Better understanding of people's responses to this type of trauma over time in wider contexts (e.g. group contexts) would help identify other factors important for resilience and recovery to be incorporated in future responses.

Agencies planning future responses in the UK could:

- Ensure there is a system to provide immediate psychological help in response to terrorist attacks, building on the experience of previous responses and on NICE guidance.

- Define and agree roles and responsibilities of all government and non-government agencies involved.

- Agree a system for collection and management of contact details of all affected people as soon as possible after an incident and including how those details would be collected under various incident scenarios (e.g. in the UK or abroad, large numbers affected in one location or spread across the country).

- Arrange data-sharing agreements between the relevant agencies, within the constraints of current legislation or by amending legislation, to facilitate rapid direct communication by the NHS with people affected.

- Prepare a plan for informing and communicating with people affected by terrorist incidents, including developing and publicizing an accessible website such as NHS Choices with information about symptoms of trauma, PTSD, depression, anxiety and other potential issues, self-care, support available and how to access mental-health services. 
- Ensure that GPs and other health professionals are adequately trained to identify PTSD and other mental-health problems and to make appropriate referrals to evidence-based treatments.

- Utilize existing networks of IAPT services and services provided by non-NHS organisations to provide timely evidence-based advice, support and treatment.

- Develop guidance for approaching problems likely to be encountered when implementing a mental-health response (e.g. assessing mental-health needs, coordinating an appropriate response, ensuring equality of access to care). 


\section{Acknowledgements}

This paper is based on independent research commissioned and funded by the NIHR Policy

Research Programme through its core support to the Policy Innovation Research Unit (Project No:

102/0001). The views expressed in the publication are those of the authors and are not necessarily those of the NHS, the NIHR, the Department of Health and Social Care, its arm's length bodies or other Government Departments.

We would like to acknowledge with thanks Dr Nika Fuchkan, who conducted the literature review, and Dr Madeleine Stevens, who carried out the cost analysis for the project. We are enormously grateful to all people who took part in the evaluation.

\section{References}

1. Neria Y, Nandi A and Galea S. Post-traumatic stress disorder following disasters: a systematic review. Psychol Med 2008; 38:467-480.

2. Boscarino JA, Galea S, Ahern J et al. Utilization of mental health services following the September 11th terrorist attacks in Manhattan, NYC. Int J Emerg Ment Health 2002; 4:143-155.

3. Ehlers A, Gene-Cos N and Perrin S. Low Recognition of Posttraumatic Stress Disorder in Primary Care. London J Prim Care (Abingdon) 2009; 2:36-42. PMCID: PMC3695460.

4. Brewin CR, Scragg $P$, Robertson $M$, et al. Promoting mental health following the London bombings: A screen and treat approach. J Trauma Stress 2008; 21:3-8. doi:10.1002/jts.20310.

5. Vandentorren S, Pirard P, Sanna A, et al. Healthcare provision and the psychological, somatic and social impact on people involved in the terror attacks in January 2015 in Paris: Cohort study. Br J Psychiatry 2018; 212:207-214. doi:10.1192/bjp.2017.63 
6. North Atlantic Treaty Organisation (NATO)/Euro-Atlantic Partnership Committee (EAPC). Annex 1 to APC(JMC)N(2008)0038. Psychosocial Care for People Affected by Disasters and Major Incidents: A Model for Designing, Delivering and Managing Psychosocial Services for People Involved in Major Incidents, Conflict, Disasters and Terrorism. Brussels, Belgium: NATO/EAPC, 2009.

7. Bisson JI, Tavakoly B, Witteveen AB, et al. TENTS guidelines: Development of post-disaster psychosocial care guidelines through a Delphi process. Br J Psychiatry 2010 Jan;196:69-74. doi: 10.1192/bjp.bp.109.066266.

8. Department of Health, Emergency Preparedness Division. NHS Emergency Planning Guidance: Planning For The Psychosocial and Mental Health Care of People Affected by Major Incidents and Disasters: Interim National Strategic Guidance. UK: Department of Health, 2009.

9. Te Brake H, Duckers M, De Vries M, et al. Early psychosocial interventions after disasters, terrorism, and other shocking events: guideline development. Nurs Health Sci 2009; 11:336-343. doi:10.1111/j.1442-2018.2009.00491.x.

10. Te Brake $\mathrm{H}$ and Dückers $\mathrm{M}$. Early psychosocial interventions after disasters, terrorism and other shocking events: is there a gap between norms and practice in Europe? Eur J Psychotraumatol 2013; 4. doi:10.3402/ejpt.v4i0.19093.

11. Brewin CR, Rose $S$, Andrews B, et al. Brief screening instrument for post-traumatic stress disorder. Br J Psychiatry. 2002 Aug;181:158-62. https://doi.org/10.1192/bjp.181.2.158

12. Kroenke K, Spitzer RL and Williams JB. The PHQ-9: validity of a brief depression severity measure. J Gen Intern Med 2001 Sep;16:606-13. https://doi.org/10.1046/j.15251497.2001.016009606.x 
13. Bush K, Kivlahan DR, McDonell MB, et al, for the Ambulatory Care Quality Improvement Project ACQUIP. The AUDIT Alcohol Consumption Questions (AUDIT-C): An Effective Brief Screening Test for Problem Drinking. Arch Intern Med 1998;158:1789-1795. doi:10.1001/archinte.158.16.1789

14. Gobin M, Rubin GJ, Albert I, et al. Outcomes of Mental Health Screening for United Kingdom Nationals Affected by the 2015-2016 Terrorist Attacks in Tunisia, Paris, and Brussels. J Trauma Stress 2018 Aug;31:471-479. doi: 10.1002/jts.22317.

15. Beecham J and Knapp M. Costing psychiatric interventions. In: Thornicroft G (ed). Measuring Mental Health Needs 2nd ed., Gaskell, Bideford, 2001, pp.200-24.

16. National Institute for Health and Care Excellence. Post-Traumatic Stress Disorder: Management. London: National Institute for Health and Care Excellence. 2005.

17. Brewin CR, Fuchkan N and Huntley Z. Evaluation of the NHS Trauma response to the London bombings. Final report to the Department of Health Forensic Mental Health R \& D programme. London, UK: University College London, Department of Clinical, Educational and Health Psychology, 2009.

18. Brewin CR, Fuchkan N and Huntley Z, et al. Outreach and screening following the 2005 London bombings: Usage and outcomes. Psychol Med 2010; 40:2049-57. doi:10.1017/S0033291710000206

19. Fuchkan N. Burden of posttraumatic stress disorder - health, social and economic consequences of exposure to the 2005 London bombings. PhD thesis. London, UK: London School of Economics and Political Science, 2015.

20. King N. Doing template analysis. In Symon G and Cassell C (eds.) Qualitative Organizational Research: Core Methods and Current Challenges. London: Sage, 2012: pp426-450. 
21. National Audit Office. Review of the Experiences of United Kingdom Nationals affected by the East Indian Ocean Tsunami. November 2006. www.nao.org.uk/wp-

content/uploads/2006/11/Review Tsunami Experiences.pdf [Accessed: 20 February 2017] 